\title{
La independencia del Banco Central y el caso de El Salvador
}

Este informe se divide en tres partes. En la primera se analizan aspectos relacionados con la función de la política monetaria. La segunda se relaciona con la función institucional del Banco Central y su independencia, y en la tercera se analiza el caso de El Salvador.

\section{La función de la política monetaria}

La única meta de la politica monetaria debería ser lograr y mantener una inflación baja y estable. Especialmente en una economía pequeña y abierı, la polftica monetaria no puede y no deberla hacer nada más. Es más, la existencia de metas múltiples en la política monetaria desestabiliza las expectativas, y hace que el logro de una tasa de inflación estable sea más difficil.

La mejor manera de ilustrar el punto anterior es analizar lo que no puede lograr una política monetaria.

(a) La política monetaria no puede permanentemente ni tampoco a mediano plazo reducir el desempleo o aumentar el crecimiento. Aunque los precios y los salarios sean fijos o rígidos, se ajustarán a la política monetaria expansionista, de manera que la expansión se traducirá (más pronto que más tarde) exactamente en mayor inflación.

(b) En una pequeña economía abierta, la función de la política monetaria como estabilizador de resultados es muy limitado o inexistente. En una economía grande, como la de Estados Unidos, la política monetaria se puede utilizar hasta cierto punto con fines anticíclicos, es decir, que la política monetaria se puede volver menos estricta y los intereses se pueden reducir durante la desaceleración de los ciclos económicos y aumentar durante las expansiones. Sin embargo, en una economía muy pequeña y abierta, las tasas de interés están 
demasiado influidas por los factores internacionales, como para que puedan ser fácilmente manejables por los políticos para propósitos nacionales.

(c) La política monetaria no puede utilizarse para financiar el déficil presupuestario. La impresión de moneda y los préslamos gubernamentales del Banco Central para financiar déficits fiscales persistentes, son el camino hacia una inflación muy alta. La experiencia latinoamericana, especialmente el caso de Argentina, muestra que la utilización del impuesto inflacionario para financiar los déficits conduce a la hiperinflación. También lo muestra la experiencia de varios países europeos después de las dos guerras mundiales. El impuesto inflacionario no aumenta monotónicamente con la tasa de inflación. Por el contrario, por encima de tasas relativamente bajas de inflación permanece constante 0 incluso disminuye. Eso se debe a que la demanda de dinero y las expeclativas reaccionan a la alta inflación real y prevista. Así, ante altos niveles de inflación, hasta los beneficios fiscales del impuesto inflacionario se pierden, sin siquiera mencionar todos los otros costos de la inflación.

(d) La política monetaria no puede y no deberá ser utilizada para distorsionar permanentemente el tipo de cambio real. Esta variable es determinada, en última instancia, por las fuerzas del mercado, tales como la productividad relativa, el costo unitario, de la mano de obra, etc. La política monetaria puede influir en el tipo de cambio nominal, el cual, a su vez, a corto plazo, puede influir en el tipo real pero sólo a corto plazo.

¿Cuáles son las evidencias empíricas de los efectos de la inflación sobre el crecimiento? Las tasas de inflación moderadas y aún persistentes (hasta, digamos, cerca del 10 al 20 por ciento anual) no son perjudiciales, aunque tampoco son beneficiosas en sí mismas. Sin embargo, por lo menos dos argumentos sugieren que incluso hasta la inflación moderada debe evitarse. El primer argumento es que existe una asociación positiva entre el nivel y la variación de la inflación. Así, pues, mientras un nivel constante de inflación moderada podría no ser perjudicial, un promedio más alto de inflación también significaría una variación más alta de la inflación. Un variación alta ciertamente es perjudicial, ya que hace que la inflación sea menos predecible y desestabilice las expectativas y la planificación económica. El segundo es que las tasas moderadas de inflación, probablemente más que las tasas bajas, degeneren en alıa inflación o incluso en hiperinflación.

La reducción de la inflación no es necesariamente costosa en términos de pérdida de producto, ni siquiera a corto plazo. Esta observación contrasta con la sabiduría convencional que dice que las estabilizaciones son costosas a corto plazo pero beneficiosas a mediano plazo. Tanto en los países en desarrollo como en los países de la OCDE, las evidencias sugieren, en cambio, que una estabilización monetaria y fiscal bien diseñada no necesita ser costosa ni a corto ni a mediano plazos. 
En resumen, la única meta que la política monetaria deberá tener es lograr y mantener una inflación baja, definida como, digamos, una tasa de inflación del 2 al 3 por ciento anual. Se necesitan dos pilares para lograr esta meta. Uno de ellos es el saldo de cuentas fiscales. Los déficits persistentes son monetizados y generan inflación, pero si no lo son, implican elevadas tasas de interés con efectos económicos adversos. El segundo pilar es la especificación del objetivo de la inflación, como meta única y específica del Banco Central. El último deberá tener libertad para poder escoger los instrumentos monetarios más apropiados para lograr el nivel deseado de inflación. En la siguiente sección se analiza la función del Banco Central y su independencia para lograr las metas inflacionarias.

\section{Independencia del Banco Central: teoría y evidencias}

El Banco Central deberá tener como única y exclusiva mesa inflacionaria, un 2 a un 3 por ciento anual. El Banco Central deberá ser independiente y deberá poder escoger los instrumentos monetarios necesarios para lograr la meta inflacionaria.

En primer lugar, es importante señalar que este arreglo no implica "un déficit democrático". De hecho, el electorado, a través de sus representantes, escoge la meta inflacionaria. El Banco Central es independiente, en el sentido de que el gobiemo no está involucrado en el manejo de la política monelaria, pero la meta del Banco se escoge democráticamente. Una vez más, esta meta tiene que ser un objetivo inflacionario del 2 al 3 por ciento.

La razón por la cual es imporante destacar la importancia de la independencia del Banco Central, es que los gobiemos tienen incentivos para abandonar los objetivos inflacionarios si pueden controlar las operaciones diarias de la política monetaria. En primer lugar, los políticos tienen incentivos para utilizar políticas monetarias expansionistas con el fin de lograr estimular, a corto plazo, la actividad económica. Estas intenciones, sin embargo, sólo logran a muy corto plazo en materia de producto y aumentan pernanentemente la inflación, desestabilizando las expectativas. En última instancia, los resultados en materia de producto son muy pequeños y transitorios y el costo inflacionario es alto y duradero ${ }^{2}$.

En segundo lugar, varios sesgos políticos hacen que los políticos se muestren propensos a mantener déficits excesivos ${ }^{3}$. Con grandes déficits presupuestarios, el gobierno tiene incentivos para abandonar la estabilidad monetaria y utilizar el impuesto inflacionario para recaudar ingresos y mantener tasas de interés más bajas. Este escenario tiene mayor posibilidad de materializarse cuando el gobierno no está dispuesto o no es capaz de recaudar impuestos, por ejemplo, en el caso de una difundida evasión. Desde el punto de vista de los políticos, el impuesto inflacionario, a corto plazo, tiene ciertas ventajas sobre otras más explícitas de tributación. El impuesto es un impuesto "oculto", y muchas veces 
quienes lo pagan no están conscientes de sus mecanismos. Por tal razón, políticamente es menos costoso utilizar un impuesto "oculto" en lugar de aumentar las formas tributarias explícitas, como el impuesto sobre la renta o el impuesto al valor agregado. También vale la pena mencionar que el impuesto inflacionario perjudica de manera desproporcional a quienes poseen activos nominales, como efectivo o bonos denominados en moneda nacional. Estos típicamente son personas pobres y de clase media baja. Los ricos pueden escapar con más facilidad al impuesto inflacionario utilizando varias coberturas, como activos reales, moneda extranjera y valores que se mantienen en el extranjero. Como se señaló antes, los intentos por monetizar los déficits presupuestarios persistentes, a menudo han degenerado en hiperinflaciones.

En tercer lugar, los políticos podrían utilizar la política monelaria de manera oportunista en los años de elecciones, para aumentar sus propias posibilidades electorales o las de de su partido4. Este comportamiento oportunista puede implicar el aumento de la oferta de dinero en los años electorales, con el fin de lograr una expansión temporaria o aumentar el crédito a cierlos departamentos gubemamentales clave, o financiar proyectos públicos que tengan una motivación electoral. Exisle una numerosa literatura sobre estos temas que hace énfasis especial en los países de la OCDEs.

Por último, si la política monetaria está bajo el control directo del gobiemo, los cambios gubernamentales de la izquierda a la derecha o viceversa, estarían asociados con grandes fluctuaciones en la política monetaria. Estos cambios conducirían a expectativas inciertas acerca de las políticas futuras, y dificultarían la planificación y la inversión ${ }^{n}$.

Por todas estas razones, un Banco Central independiente, comprometido con una meta inflacionaria, resulta mucho mejor que un arreglo institucional en el que el gobiemo tenga el control direclo de la política monetaria. Un Banco Central independiente dificultaría o imposibilitaría que el gobiemo distorsionara la política monetaria en las formas que se han descrito con anterioridad. Sin embargo, en este punto es importante subrayar qué es lo que la independencia del Banco Central puede y no puede lograr. Si un gobierno está firmemente determinado a aplicar cualquiera de las políticas subóptimas descritas anteriormente, de manera eventual lo va a lograr aunque sea eliminando la independencia del Banco Central si fuera necesario, y abandonando la meta inflacionaria. Todo lo que puede pedirse y esperarse de una ley que establezca la independencia del Banco Central, es que los gobiemos que quieran seguir el lipo de políticas ya descritas les sea más difícil hacerlo, por ejemplo, porque resulta difícil eliminar la independencia del Banco Central. Puesto que las leyes más difíciles de cambiar son las constitucionales, es una buena idea incluir la mela inflacionaria como la única meta del Banco Central en la propia Constitución. Esta también debería incluir disposiciones que garanticen la independencia política del Banco. 
¿Cuáles son las evidencias empíricas del efecto de la independencia del Banco Central? El primer artículo que enfoca directamente este asunto es un trabajo inédito de Bade y Parkin (1982). Estos autores consideran una muestra de varios países de la OCDE y clasifican el grado de independencia de sus bancos centrales. Los factores que consideraron fueron la duración del nombramiento del presidente del Banco; si los funcionarios gubernamentales podían ocupar cargos en el directorio del Banco; si éste tenía un sólo objetivo inflacionario, etc. Los autores encontraron que el índice de independencia del Banco Central estuvo asociado con la inflación promedio en los años sesenta y setenta: cuanto más independientes eran los bancos centrales, menor era la inflación. En un trabajo preparado a finales de los años ochenta (Alesina, 1988), revisé el índice preparado por Bade y Parkin y aumenté el número de países cubiertos, incluyendo un mayor número de países de la OCDE. En ese estudio confirmé sus resultados, es decir, que una mayor independencia está asociada con una menor inflación.

Grilli, Masciandaro y Tabellini (1990) prepararon olro índice que era superior al mío, ya que tomaron en cuenta más factores y dividieron el índice en dos componentes: independencia política e independencia económica. El primer componente se refiere a los procedimientos de nombramiento, la ley del Banco Central, etc. El componente económico considera, en particular, si se requiere que el Banco Central financie o no los déficits presupuestarios, si puede prestar directamente al gobierno, si el gobierno puede solicitar al banco que mantenga bajas las tasas de interés, etc $^{7}$. Estos autores también descubrieron que su índices de independencia estaban fuertemente asociados con el promedio y la variación de la inflación. Ambos eran menores en países con bañcos centrales más independientes. Según este índice, los bancos centrales más independientes eran los de Alemania, Suiza y Estados Unidos ${ }^{8}$.

Alesina y Summers (1993) analizan si la menor inflación lograda por la independencia del Banco Bentral se produce a costa de un desempleo más alto, un menor crecimiento o una mayor variación de estas variables. Utilizaron un promedio de lodos los índices disponibles de independencia y hallaron que si bien la inflación era más baja y más estable, no habían costos obvios en términos de las variables económicas "reales" (en especial, el desempleo y el crecimiento). Otros autores han confirmado estos resultados, utilizando diversos índices alternativos en países de la OCDE.

En una serie de trabajos, Cukierman y asociados" han extendido la muestra de países más allá del grupo de la OCDE y han considerado una muestra de alrededor de 80 países de la OCDE y en desarrollo. Los resultados son muy interesantes. Con respecto a los países de la OCDE, esta investigación, basada en un índice nuevo y cuidadosamente preparado, confirma los resultados anteriores. Los resultados en los países en desarrollo, en cambio, son diferentes. Las disposiciones de la ley que rige la situación institucional del Banco Central no 
están relacionadas con la inflación, ni con ninguna otra variable económica de interés. En cambio, estos autores encontraron que la frecuencia del reemplazo del presidente del Banco Central estaba fuertemente asociada con la inflación. El reemplazo más usual está asociado con una mayor inflación y la causalidad funciona en ambas direcciones, es decir, una inflación más alta conduce a mayores reemplazos en el Banco Central, y los reemplazos frecuentes desestabilizan la inflación.

La diferencia en los resultados observados en los dos grupos de países es bastante interesante, y es posible imaginarse dos explicaciones relacionadas. En primer lugar, en el período de muestra considerado en estos estudios (que abarcan desde los años cincuenta a fines de los ochenta) muchos países del grupo en desarrollo no eran democráticos. En una dictadura, casi por definición, el respelo a la letra de la ley es imperfecto en el mejor de los casos. En segundo lugar, podría decirse que parte del proceso de desarrollo es el fortalecimiento de las instituciones. Los países con menor desarrollo económico también tienen un menor desarrollo instilucional, de manera que, de nuevo, la letra de la ley podría ser menos taxativa. En cualquier caso, estos resultados llevan al tema crítico de la reforma de la ley del Banco Central que, aunque es necesaria, podría no asegurar por sí misma el logro de una independencia real, sino que una independencia solamente "de jure" que sólo se aplica de manera imperfecta en la práctica ${ }^{\prime \prime \prime}$.

Los resultados observados en los países en desarrollo ha llevado a algunos autores (por ejemplo, Barro, 1997) a argumentar que la independencia del Banco Central es irrelevante. Desde el punto de vista de la política, esta conclusión es incorrecta. Puede ocurrir que, en ciertos países, especialmente con bajos niveles de institucionalización y de desarrollo económico, la independencia consagrada por la ley podría no ser suficiente para garantizar la independencia en la práctica. Sin embargo, lo mejor que podría esperarse es que a medida que se desarrolla como institución, un banco central independiente podría pasar a ser relevante y afirmar su propia autonomía.

\section{El caso de EI Salvador}

¿Cuáles son las implicaciones del análisis anterior para el caso de El Salvador? Quisiera organizar mis comentarios alrededor de ciertos puntos.

(a) Para poder mantener la estabilidad macroeconómica, la única y exclusiva meta del Banco Central Salvadoreño debería ser inflacionaria. Esta única meta debería estar especificada en la Constitución.

En la actualidad, se ha propuesto una reforma constitucional que prohibe que el Banco Central preste fondos al gobiemo. La inclusión de esta disposición en la Constitución constituye un paso importante en la dirección correcta. Una dis- 
posición más completa incluiría una declaración muy clara en un artículo de la Constitución, en el que se estableciera que la única meta del Banco Central es mantener "la estabilidad de precios", definida como una inflación muy baja.

(b) La Constitución debería garantizar la independencia del Banco Central en el logro de esta meta.

Es importante que el concepto de independencia del Banco Central esté enfatizado explícitamente en la Constitución, porque en el caso de un conflicto entre un Banco Central que trata de lograr su meta de estabilidad de precios y el gobiemo, el banco puede apelar a la Constitución. Si el gobiemo tiene que violar la Constitución para invalidar una disposición del Banco Central, su costo político podría ser muy alto, de manera que algunos gobiemos podrían decidir no hacerlo.

(c) Los integrantes del directorio del Banco Central deberian ser designados por el gobierno.

Actualmente, los representantes de la comunidad empresarial que incluyen representantes del sector bancario, ocupan cargos en el directorio del banco central. Si bien existen ciertas disposiciones especiales orientadas a la limitación de los conflictos de intereses, éstos no siempre pueden eliminarse por completo, en especial en el caso de los representantes del sector bancario. En términos generales, es importante subrayar la división y la separación del papel de la comunidad empresarial del Banco Central como entidad pública. Además del argumento del conflicto de intereses, la eliminación de los banqueros del directorio del Banco Central también sirve un propósito político muy importante. El apoyo político en favor de la independencia del Banco Central y de la meta de baja inflación podría ser mayor, incluso en los círculos de la izquierda si el público percibe que el Banco Central realmente desempeña una función pública y no está influido indebidamente por un grupo reducido de la población, como la comunidad empresarial y el sector de la banca en particular.

(d) Los nombramientos en el directorio deberian ser escalonados y los períodos de duración del presidente del banco no deberían coincidir con el período del Presidente de la República.

Si cada nuevo presidente nombra a un nuevo gobernador del Banco Central y un nuevo directorio ejerce un control completo sobre el personal que dirige el banco. En cierto sentido, el presidente del Banco Central es un ministro del gobierno: el ministro encargado de la política monetaria. Si bien la meta inflacionaria debería limitar la influencia del gobierno y del presidente en la política monetaria, aun así un vínculo tan directo entre el Presidente de la República y el presidente del Banco Central no asegura una suficiente separación de funciones. Esto podría potencialmente tener varias consecuencias negativas. En primer lugar, la política monetaria podría cambiar de manera abrupta cuando se 
nombre a un nuevo gobierno y el órgano directivo del Banco Central sea reemplazado por completo. En segundo lugar, el presidente del Banco Central podría sentirse "obligado" a ayudar al partido del presidente que lo nombró, cuando sus nombramientos estén expirando y se acerquen las nuevas elecciones.

Ciertamente es preferible que el período del presidente del Banco Ceniral sea más largo que el del Presidente de la República o, por lo menos, de igual duración, pero sin que coincidan las fechas de nombramiento. Igualmente, los nombramientos de los miembros del directorio deberían ser escalonados, en diferentes fechas, para que ningún gobierno pueda nombrar de inmediato a todos los miembros del directorio. Esta estructura de nombramientos garantiza más estabilidad en la dirección de la polílica monelaria y puede reducir las fluctuaciones de política y estabilizar las expectativas. Vale la pena señalar que esta estructura y esta programación de los nombramientos se aplican en Estados Unidos.

(e) La supervisión del sector bancario deberia asignarse al Banco Central.

El aspecto más importante de la supervisión bancaria es que el supervisor no sea "capturado" por los supervisados. Un Banco Central fuertemente independiente podría ser una institución menos susceptible de ser "capturada". Dada esta función del Banco Central es aún más crítico, como se señalaba anteriormente, que los representantes del sector bancario estén excluidos por completo del órgano directivo del Banco Central. De otra manera, quienes deben ser controlados pasan a ser los que controlan.

Antes de concluir, es necesario destacar la conexión que existe entre la independencia del Banco Central, la estabilidad monetaria, la baja inflación y la función de la política fiscal. Como se decía con anterioridad, un pilar crítico para la estabilidad monetaria es el equilibrio fiscal. Los déficits presupuestarios persistentes son inconsistentes con la estabilidad monetaria y la existencia de tasas de interés razonablemente bajas. Por tal razón, el gasto público no puede financiarse emitiendo bonos, sino con ingresos fiscales. Esta consideración es en particular crítica para El Salvador, un país que necesita una mejor infraestructura (parte de la cual fue destruida por la guerra civil) y una mejor educación pública".

En consecuencia, resulta crucial para El Salvador aumentar los ingresos para poder mantener el equilibrio fiscal y lograr una inflación baja y estable. Los impuestos, incluido el impuesto al valor agregado, no pueden y no deberán reducirse ni cortarse. Por el contrario, el gobiemo debería realizar un esfuerzo por recaudar más ingresos. El impuesto sobre la renta es ampliamente evadido y debería realizarse un mayor esfuerzo por aumentar su recaudación.

Enero 29, 1999. 


\section{Referenclas bibllogriffcas}

Alesina, A., "Macroeconomics and politics", NBER Macroeconomic Annual, 1988.

Alesina, A. y Perotti, R., "The political economy of budget deficits", IMF Staff Papers, 1995.

Alesina, A.; Roubini, N. y Cohen, G., Political Cycles and the Macro economy, MIT Press, 1997.

Alesina, A. y Summers, L., "Central Bank Independence and macroeconomic performance", Journal of Money Credit and Banking, 1993.

Bade, R. y Parkin, M., "Central Bank laws and monetary policy", inédito, 1982.

Barro, R., Determinants of Economic Growth, MIT Press, 1997.

Barro, R. y Gordon, D., "Rules, discretion and reputation in a model of monetary policy", Journal of Monetary Economics, 1983.

Cukierman, A., Central Bank Strategy. Credibility and Independence, MIT Press, 1993.

Grilli, V.; Masciandaro, D. y Tabellini, G., "Political and monetary institutions and public financial polices in the industrial countries", Economic Policy, 1990.

Kydland, F. y Prescott, E., "Rules rather than discretion: the inconsistency of oplima plans", Journal of Political Economics, 1977.

Posen, A., "Declarations are not enough: financial sector sources of central bank independence. ", NBER Macroeconomic Annual, 1995

\section{Notas}

1. Este documento fue preparado después de una visita a El Salvador en junio de 1997, la cual fue subvencionada por la misión de la USAID. Deseo agradecerle a Nolvia Saca por sus comentarios en un borrador anterior.

2. En términos técnicos, a este problema se le conoce como el problema de la "inconsistencia en el tiempo" de la política monetaria, y ha sido expuesto por Kydland y Perescott (1977) y Barro y Gordon (1983).

3. Véase en Alesina y Perotti (1995) un análisis de los modelos políticos de déficits presupuestarios.

4. Este argumento se aplica también en aquellos países en los que el presidente no puede ser reelegido por más de un período; de hecho, un presidente estaría interesado en el futuro de su partido.

5. Véase, en particular, Alesina, Roubini y Cohen (1997).

6. Sobre este punto, véase Alesina, Roubini y Cohen (1997).

7. No todos los países mostraban valores similares en ambos componentes del índice. Por ejemplo, Italia logró altas calificaciones en materia de independiencia política, pero muy bajas en términos de independencia económica.

8. Este trabajo empírico se llevó a cabo antes de la reforma radical del Banco 
Central en Nueva Zelandia, que hizo que el Banco Central de este país fuera uno de los más independientes del mundo.

9. Los resultados de esta investigación se resumen en Cukierman (1993).

10. Un aspecto interesante que no hemos explorado es qué determina la selección de leyes de la banca central, y si ésa es la razón por la que los diferentes países escogen distintos niveles de independencia del Banco Central. Véase un análisis sobre este tema en Posen (1995).

11. Las comparaciones internacionales revelan que los logros educacionales y las tasas de inscripción en escuelas secundarias en El Salvador y Centroamérica son, en general, bastante bajas, incluso al tener en cuenta los niveles de ingresos de los países. 